\title{
Caracterización de la movilidad en bicicleta en el Campus Universitario Rodrigo Facio, Universidad de Costa Rica
}

\author{
Characterization of bicycle mobility on the campus Rodrigo Facio, University of Costa \\ Rica
}

\author{
Henry Hernández Vega \\ Laboratorio Nacional de Materiales y Modelos Estructurales \\ Universidad de Costa Rica, henry.hernandezvega@ucr.ac.cr
}

Verónica Lezama Jara

Laboratorio Nacional de Materiales y Modelos Estructurales

Universidad de Costa Rica. vlezamaj92@gmail.com

Mónica Gutiérrez Barboza

Laboratorio Nacional de Materiales y Modelos Estructurales

Universidad de Costa Rica. monigb17@gmail.com

Fecha de recepción: 26/09/2016. Fechas de reenvíos: 11/11/2016 -15/12/2016,

Fecha de aprobación: 23/02/2017. Fecha de publicación: 19/04/2017.

\begin{abstract}
Resumen: El presente artículo tiene como objetivo caracterizar a las personas que viajan en bicicleta hacia la Universidad de Costa Rica por medio de un análisis de los resultados de la Encuesta de Transporte realizada por el Programa de Infraestructura del Transporte (PITRA) del Laboratorio Nacional de Materiales y Modelos Estructurales de la Universidad de Costa Rica en el 2013. Con los datos de los viajes en bicicleta se determinó que un $42 \%$ de los usuarios encuestados que asisten a la universidad en bicicleta lo hacen desde distancias que se ubican entre los dos y los cinco kilómetros. Además, un $62 \%$ de quienes utilizan este medio de transporte tarda 30 minutos o menos en realizar el recorrido diario hacia la Universidad. Existe una diferencia por sexo respecto al uso de la bicicleta dado que el $81 \%$ de las personas ciclistas corresponde a hombres y el $19 \%$ a mujeres. La ausencia de espacio exclusivo para bicicletas es uno de los principales problemas experimentados por los ciclistas y las ciclistas. Vehículos transitando a altas velocidades, la presencia de vehículos pesados y la conducción nocturna sin presencia de iluminación en la vía son factores que influyen en la percepción de seguridad que tienen las personas ciclistas en sus itinerarios. Finalmente, la existencia de infraestructura exclusiva para ciclistas en la vía, la implementación de espacios de parqueo y educación para los conductores y las conductoras se consideran como las principales incentivos para el uso de la bicicleta.
\end{abstract}


Palabras Claves: transporte urbano; ciclo-movilidad; actividad física; política de salud en el transporte.

\begin{abstract}
This paper aims at characterizing bicyclists commuting to the University of Costa Rica, by analyzing the results of the Transportation Survey conducted in 2013 by the Transport Infrastructure Program (PITRA) of the National Laboratory of Structural Models and Materials of the University of Costa Rica. The collected data determined that $42 \%$ of respondents commute between two and five kilometers. In addition, $62 \%$ take 30 minutes or less to get to the university every day. There is a difference on bicycle use per sex given that $81 \%$ of cyclists are men and $19 \%$ are women. The lack of exclusive spaces for bicycles is one of the main problems experienced by cyclists. Vehicles at high speed, heavy vehicles on the road, and the lack of road lighting at night are some of the factors that influence the perception of cyclist safety. Finally, exclusive cycling infrastructure, bicycle parking spaces and driving education are considered the main incentives to ride a bicycle.
\end{abstract}

Keywords: Urban transport; cycle-mobility; physical activity; transport health policy.

\title{
Introducción
}

El presente estudio se realiza en el contexto del campus universitario de la Universidad de Costa Rica (UCR), específicamente en la Sede Rodrigo Facio; esta se encuentra en San Pedro de Montes de Oca en la provincia de San José. Cuenta con una extensión de 52,5 hectáreas entre sus dos principales áreas, y posee una población estudiantil activa de aproximadamente 40000 personas distribuidas entre su sede central y las sedes regionales.

De acuerdo con Leandro, Molina y Riba (2013), el 60 \% del espacio total disponible en la Sede Rodrigo Facio es utilizado como parqueo. Indican que únicamente el $23 \%$ de las personas usuarias del Campus utilizan el vehículo como medio de transporte, de modo que muy pocas personas utilizan la mayoría del espacio para movilizarse, situación que tiende a agravarse ya que, según datos de la Unidad de Promoción de la Salud de la UCR, aproximadamente el $70 \%$ de la población universitaria no realiza ningún tipo de actividad física.

La UCR ha buscado generar estrategias que en conjunto logren cambiar no solo la realidad que enfrenta en temas de movilidad y disposición del espacio, sino también enfocadas en asegurar la accesibilidad en el Campus y el uso de la bicicleta; como parte de estas se ha implementado el Plan de mejoramiento para la movilidad y el transporte en la Ciudad Universitaria, ya que se "pretende convertir su campus en un espacio armónico, amigable y atractivo con el objetivo de promover la movilidad activa y una visión de universidad saludable" (Universidad de Costa Rica, 2012). Entre algunos aspectos que se contemplan, está el principio de la vía compartida, "donde se pretende ofrecer al peatón y al ciclista un espacio seguro para su movilidad, donde 
sean ellos quienes tengan la prioridad al transitar dentro del campus universitario. El objetivo es que paulatinamente se vaya creando conciencia y mejor aceptación de la bicicleta dentro de la Universidad" (Burgos \& Marín, 2014).

Es importante acotar que el aumento en el tamaño de la flota vehicular nacional, ha tenido un impacto directo en el Campus Rodrigo Facio de la UCR, pues existe una importante presión por más espacios para vehículos. Toor y Havlick (2004) indican que la mayoría de los campus han sido diseñados como recintos peatonales, pero se ha caído en una cultura que fomenta utilizar el automóvil en cada oportunidad; esto provoca una presión sobre las autoridades de las universidades para desarrollar mayores espacios de parqueos y facilidades para los automóviles, de modo que se abandone el desarrollo de la infraestructura que estimule el transporte no motorizado.

Las universidades son capaces de influir en el comportamiento de viaje (Millard-Ball, Siegman \& Tumlin, 2004), y son lugares privilegiados para comunicar la sostenibilidad (Balsas, 2003), de modo que deben de trabajar de una manera activa en generar insumos que logren suscitar políticas que promuevan una movilidad que se traduzca en personas más saludables; una buena opción es desarrollando planes para el transporte en bicicleta y estudios como los presentados en diferentes universidades (Alta Planning + Design, Kimley-Horn and Associates, Inc., 2012; Kaplan, 2015; Portland State University, 2011; University of North Carolina at Greensboro, 2008; University of California Berkeley, 2006). Estos planes tienen el objetivo de aumentar la cantidad de estudiantes, personal docente y administrativo que utilizan la bicicleta a través de intervenciones de la infraestructura, programas de educación, promoción y regulación, entre otros. Los estudios permiten entender mejor los patrones de movilidad e identificar oportunidades para incentivar la movilidad activa.

Existe una relación entre las políticas de movilidad implementadas en las ciudades y la salud de sus habitantes. De acuerdo con Wen, Orr, Millet y Rissel (2006), las personas que manejan hacia el trabajo tienen niveles de actividad física inferiores a los recomendados; por otro lado, las que utilizan la bicicleta y el transporte público para ir al trabajo tienen menos probabilidades de tener sobrepeso o padecer de obesidad. Aparte de la reducción en los niveles de obesidad relacionados con la movilidad activa, la Organización Mundial de la Salud (2009), así como Pucher y Dijkstra (2003), indican que las disminuciones en emisiones, debido al uso de transporte no motorizado, generan beneficios en la salud respiratoria de la población, se logra disminuir el congestionamiento vial, se ahorra energía, se reduce la contaminación y con esto se logra una mejor conservación del ambiente.

El empleo de diferentes medios para el desplazamiento como son el caminar, el uso de la bicicleta y el uso del transporte público tienen efectos positivos tanto en el medio ambiente como en la salud de la población. El sobrepeso y obesidad en niños, niñas y adolescentes están asociados, 
entre otras razones, con la falta de medios de transporte activos para viajar a la escuela (Duncan et al, 2011).

En los últimos años se han generado, en diferentes ciudades, nuevas políticas orientadas "hacia modelos y estrategias de desarrollo urbano en donde el caminar, usar la bicicleta y el transporte público sean los elementos alrededor de los cuales se genera el desarrollo de las ciudades" (Medina y Veloz, 2013, p. 10).

El incremento de la actividad física gracias a la movilización en bicicleta incide en la salud de la población en general. Gotschi (2011, p. 49) indica que"promover el ciclismo tiene gran potencial para incrementar la actividad física". La movilidad en bicicleta tiene grandes posibilidades cuando se combina con transporte público y con peatonalización. El objetivo central es lograr cohesionar los diferentes medios de transporte, buscando no solo diversificarlos sino también aprovechar lo mejor de cada uno. Incorporar las bicicletas y el transporte público puede mejorar el acceso, ayudar a reducir los tiempos de viaje, crear ciudades con transporte más sostenible y ciudadanos más sanos.

El "ciclismo urbano es una opción de movilidad con alto potencial para reducir algunos de los problemas de nuestras ciudades: la congestión de tráfico, la mala calidad del aire y las emisiones de gases que contribuyen al cambio climático" (Banco Interamericano de Desarrollo, 2015, p. 1). Estos beneficios se han aceptado ya en muchas ciudades latinoamericanas y es por ello que han incorporado la bicicleta en un proceso de reconstrucción de visión del espacio urbano que está dando inicio.

El uso de la bicicleta en Costa Rica es un tema que se ha venido desarrollando desde hace relativamente poco. Datos del diagnóstico del Plan Regional Urbano de la Gran Área Metropolitana de Costa Rica (PRUGAM) demostraron que para el año 2007, durante el periodo pico de la mañana solo el 1,8\% de los viajes de la Gran Área Metropolitana se realizaban en bicicleta (PRUGAM, 2008). Posterior al periodo de la recopilación de datos para el presente estudio, información del Banco Interamericano de Desarrollo (2015), plantea que para el 2014 un $2 \%$ de los viajes en el Área Metropolitana de San José se realizaban en bicicleta, donde se reportaron aproximadamente 125 mil viajes diarios en ese medio de transporte.

Según el Plan Nacional de Actividad Física y Salud 2011-2020 (Ministerio de Salud, 2010), la inactividad física en el 2009 se pudo determinar como la cuarta causa más significativa en el mundo de muertes de origen crónico degenerativo y el motivo del aumento en más de tres millones del número de muertes prevenibles. Este plan hace referencia a los bajos porcentajes de población adolescente que realiza el mínimo de actividad física recomendada. 
La Organización Mundial de la Salud (2017), indica que en el mundo una cuarta parte de los adultos y cuatro quintas partes de los adolescentes no tienen un nivel suficiente de actividad física. Hay un aumento en el sedentarismo tanto en el hogar como en el trabajo y las personas, en sus momentos de ocio, no se involucran en actividades físicas. Además, ha disminuido la actividad física por el uso de formas de transporte "pasivo".

El caso de Costa Rica no es diferente al de la mayoría de países americanos y europeos: se han identificado altos niveles de sedentarismo y obesidad, esto en todos los grupos etarios. Por ejemplo, Loría (2014), indica que la mayoría de la población estudiantil de la Sede de Occidente de la Universidad de Costa Rica tiene una vida sedentaria o con limitada actividad física, siendo más crítico para el caso de las mujeres. Sin embargo, en otros estudios, se identificó que el porcentaje de ciclistas femeninas corresponde a un $26 \%$ en Puntarenas (Espinoza, 2015), a un aproximado de 1,3\% en los distritos del suroeste de la ciudad de Cartago (Torres, 2016), y a un ocho por ciento en las ciclovías de la ciudad de Cartago (Acuña, 2015). Se vislumbra así una tendencia positiva, ya que Acuña (2015), determinó que la construcción de la nueva ciclovía de Cartago podría motivar el surgimiento de nuevas mujeres ciclistas, ya que se ha observado un crecimiento del $50 \%$ de mujeres viajando en bicicleta desde la creación de la ciclovía.

En esta línea, el presente artículo presenta los resultados del apartado referido a la movilidad en bicicleta, de la Encuesta de Transportes realizada en 2013 dirigida a estudiantes y funcionarios de la Sede Rodrigo Facio de la UCR con el objetivo de presentar y contextualizar la ciclo-movilidad en esta universidad, como parte del plan de movilidad, que busca obtener información útil para generar políticas en función del incremento de la bicicleta como medio de transporte habitual, de modo que se promueva la movilidad activa, para desarrollar un campus universitario más saludable, combinando la actividad física y el mejoramiento de los espacios dentro de la universidad. Se espera generar insumos que pueden tener una influencia directa en la definición de políticas de cambio dentro y fuera de la Universidad de Costa Rica.

El estudio permite determinar el perfil de los usuarios de bicicleta incluyendo inter alia: edad, sexo, condición laboral, tipo de usuario del campus, la frecuencia y razones de uso de la bicicleta; las rutas y distancias recorridas; los motivo para usar este medio de transporte, así como aquellas razones que les desalientan; también, los problemas y los desafíos que encuentran al movilizarse de esta manera.

El artículo aborda la caracterización y percepción de quienes viajan a la universidad en bicicleta, convirtiéndolo en una herramienta para dimensionar mejoras y medidas que permitan incentivar el uso, motivando a que la población utilice la bicicleta como un medio de transporte usual. La información aquí presentada puede ser de utilidad a los tomadores de decisiones a la hora de implementar proyectos relacionados con movilidad en bicicleta. 


\section{Metodología}

El cuestionario utilizado en este estudio se realizó en formato electrónico tomando en cuenta aspectos prácticos, especialmente considerando el bajo costo de los mismos en este formato (Díaz de Rada, 2012). Se considera que los sondeos por internet facilitan información de tanta calidad como las encuestas presenciales o telefónicas (Esquivel, González, \& Martínez, 2012). Similares resultados se han obtenido con personas jóvenes y universitarias (Heerwegh y Loosevelt, 2009).

El cuestionario fue enviado en 2013 a estudiantes y funcionarios de la UCR con el propósito de "caracterizar los viajes para lograr un mejor entendimiento de los patrones de movilidad y necesidades de las personas usuarias de las instalaciones de la UCR" (Hernández y Jiménez, 2014, p. 1).

Un total de 5323 personas ingresaron al portal de internet y contestaron por lo menos una de las preguntas planteadas. De esas 5323 personas, por medio de la aplicación de filtros, se llegó a un total de 77 personas identificadas como ciclistas, personas que frecuentemente utilizaban la bicicleta para viajar hacia la UCR al momento de aplicar el cuestionario electrónico.

Dentro de las limitaciones del sondeo aplicado, se debe aclarar que no se realizaron ajustes a la información presentada. Para poder realizar correcciones socio-demográficas es necesario contar con información detallada tanto del alumnado como del personal docente y administrativo de la UCR. Al momento de realizar el estudio no se contó con esta información.

Además, de acuerdo con Rodríguez, et al (2013), el sesgo de participación afecta la representatividad de la información recopilada. Por otro lado, Vives, Ferreccio y Marshall (2009), mencionan diferentes técnicas para corregir el sesgo de participación; sin embargo, en ciertos casos, como el de la sustitución muestral, podría generar un sesgo mayor que otras técnicas. Adicionalmente, indican que utilizar muchas variables para generar factores de ajuste podría generar una inestabilidad en los ajustes. Asimismo, Cuddeback, et al (2004), indican que realizar correcciones cuando no se conocen las características de la población no participante, puede generar dudas acerca de las conclusiones de cualquier investigación o inclusive podría empeorar la calidad de las estimaciones.

Un estudio acerca del impacto de la no-respuesta en estudios de transporte (Forsman, Gustafsson y Vadeby, 2007), reporta una calibración por no-respuesta con efectos pequeños e inconsistentes y sugiere que la tasa de respuesta podría depender del modo de transporte utilizado. 
Dada la naturaleza del sondeo y del bajo porcentaje de personas que reportan usar la bicicleta, junto con una muestra de 77 ciclistas que participaron en el estudio, no se considera necesario realizar correcciones por el riesgo de generar un mayor sesgo de los resultados. El análisis de la no participación, está más allá de los alcances del presente estudio.

Debido a la limitada información relacionada con la movilidad ciclista en el país, y especialmente en la movilidad en bicicleta hacia la UCR, los resultados mostrados corresponden a un sondeo exploratorio que pretende presentar resultados iniciales que deberán ser validados mediante estudios detallados a futuro.

Se asume que las personas participantes en el sondeo representan a la población ciclista de la UCR al momento de realizar la recopilación de información. Los autores recomiendan al lector ser críticos de este supuesto y las conclusiones de este estudio deben de ser vistas a la luz de las limitaciones expuestas en los párrafos anteriores. Los intervalos de confianza indicados en el presente documento, así como las barras de error mostradas en los gráficos corresponden a intervalos de confianza al $95 \%$.

\section{Resultados}

A continuación se presentan los resultados de las respuestas que corresponden a usuarios que utilizan la bicicleta como medio de transporte al menos dos días por semana. Estos podrían servir como insumos que permitan generar políticas y estrategias para la promoción del ciclismo en la UCR.

Sobre la generalidad de los datos recolectados, el total de 77 respuestas cumplieron los criterios de inclusión en el estudio (disponibilidad de usar una bicicleta y días a la semana que la utilizaban como medio de transporte para ir a la Universidad). De esta forma se logró determinar que aproximadamente el $1 \%$ de las personas que viajan hacia la Sede Rodrigo Facio, lo hacen en bicicleta.

El $63,6 \pm 10,7 \%$ de las personas entrevistadas tiene una edad entre 16 y 25 años, seguido por quienes tienen entre 26 y 35 años, los cuales representan un $27,2 \pm 9,9 \%$. Un $9,1 \pm 6,4 \%$ de las personas ciclistas participantes corresponden al grupo entre los 36 y los 55 años. Existe una clara predominancia masculina en el grupo de entrevistados $(80,5 \pm 8,8 \%)$.

El $83,1 \pm 8,4 \%$ de quienes fueron entrevistados son estudiantes regulares, seguido por funcionarios y funcionarias, docentes y personal administrativo (14,3 $\pm 7,8 \%$ ). Los restantes porcentajes corresponden a estudiantes realizando su proyecto de graduación, estudiantes de posgrado, visitantes y estudiantes de cursos libres. 
Respecto al nivel educativo máximo alcanzado, el 72,7 $\pm 9,9 \%$ de los entrevistados y las entrevistadas cuenta con universidad incompleta. El 16,9 $\pm 8,4 \%$ corresponde a personas con bachillerato y licenciatura universitarias y en menores porcentajes, secundaria completa, técnico y profesorado, universidad incompleta en carácter inactivo y maestría.

Con respecto a la experiencia en el uso de la bicicleta, el 58,4 $\pm 11,0 \%$ de las personas encuestadas afirmó ser experto, un $35,1 \pm 10,7 \%$ indicó tener un nivel intermedio y un $3 \%$, es principiante. El $48,1 \pm 11,2 \%$ de los usuarios afirmaron hacer uso de la bicicleta entre uno y tres días la semana, además un $41,6 \pm 11,0 \%$ dice utilizarla a diario.

El 88,3 \pm 7,2 \% de las personas entrevistadas proviene de la provincia de San José, principalmente desde los distritos de Sabanilla, San Pedro, Guadalupe, Moravia, Curridabat y Desamparados y Tres Ríos de la Unión, de Cartago. A continuación se presentan datos puntuales sobre tipos de recorridos, distancias y la percepción de los usuarios en función de motivos y problemáticas identificadas durante sus traslados.

\section{Caracterización de los viajes en bicicleta a la sede Rodrigo Facio de la UCR}

El 55,1 $\pm 11,1 \%$ de las personas entrevistadas afirmó asistir a la universidad cinco días entre semana (no se consideran los fines de semana), el 24,4 $\pm 9,6 \%$ cuatro días a la semana y el 14,1 $\pm 7,8 \%$ tres veces; el restante porcentaje indicó asistir una o dos veces a la semana.

Sobre el uso de la bicicleta, un 27,3 $\pm 10,0 \%$ de los participantes la utilizan un día a la semana; adicionalmente, un 22,1 $\pm 9,3 \%$ utilizan la bicicleta los cinco días de la semana; el restante porcentaje los usan un día en específico.

Después de la bicicleta, el autobús es el medio de transporte más utilizado por parte de los ciclistas y las ciclistas para viajar a la universidad. Por otro lado, el 49,4 $\pm 11,1 \%$ de quienes fueron entrevistados afirmó haber utilizado otro medio de transporte antes de empezar a utilizar la bicicleta de manera más frecuente; se destaca una relación entre ciclistas universitarios y el autobús. Un 17,5 $\pm 8,5 \%$ pasó de conducir un automóvil a usar la bicicleta. Las razones de uso de la bicicleta que exponen las personas entrevistadas se relacionan con que es un medio más económico y rápido, razones ambientales y de salud, y por vivir cerca de la universidad.

Sobre el tema de tiempos de viaje y kilómetros recorridos, no se encontró una diferencia considerable entre los porcentajes de viajes de ida hacia la universidad y de regreso hacia los lugares de residencia. La mayoría de las personas ciclistas universitarias tienen recorridos menores a 45 minutos (Tabla 1). 
Tabla 1

Distribución porcentual de los tiempos de viaje de los usuarios de bicicleta hacia la Universidad de Costa Rica

\begin{tabular}{ccccc}
\hline $\begin{array}{c}\text { Tiempo } \\
\text { (minutos) }\end{array}$ & $\begin{array}{c}\text { Hacia la } \\
\text { Universidad }\end{array}$ & $\begin{array}{c}\text { Intervalo Confianza } \\
95 \%\end{array}$ & $\begin{array}{c}\text { Desde la } \\
\text { Universidad }\end{array}$ & $\begin{array}{c}\text { Intervalo Confianza } \\
95 \%\end{array}$ \\
\hline$<15$ & $27,3 \%$ & {$[17,3 \%-37,3 \%]$} & $26,0 \%$ & {$[16,2 \%-35,8 \%]$} \\
$15-30$ & $35,1 \%$ & {$[24,4 \%-45,8 \%]$} & $31,2 \%$ & {$[20,9 \%-41,5 \%]$} \\
$30-45$ & $20,8 \%$ & {$[11,7 \%-29,9 \%]$} & $24,7 \%$ & {$[15,1 \%-34,3 \%]$} \\
$45-60$ & $10,4 \%$ & {$[3,6 \%-17,2 \%]$} & $10,4 \%$ & {$[3,6 \%-17,2 \%]$} \\
$60-75$ & $2,6 \%$ & {$[0 \%-6,2 \%]$} & $5,2 \%$ & {$[0,2 \%-10,2 \%]$} \\
$75-90$ & $1,3 \%$ & {$[0 \%-3,8 \%]$} & $0 \%$ & - \\
$90-105$ & $0 \%$ & - & $0 \%$ & - \\
$105-120$ & $2,6 \%$ & {$[0 \%-6,2 \%]$} & $1,3 \%$ & {$[0 \%-3,8 \%]$} \\
$>120$ & $0 \%$ & - & $1,3 \%$ & {$[0 \%-3,8 \%]$} \\
\hline
\end{tabular}

Fuente: Encuesta de Transporte-UCR, 2013.

Asimismo, la mayoría de los viajes se realizan a distancias menores de 10 kilómetros al campus, predominando viajes entre dos y diez kilómetros (Figura 1).

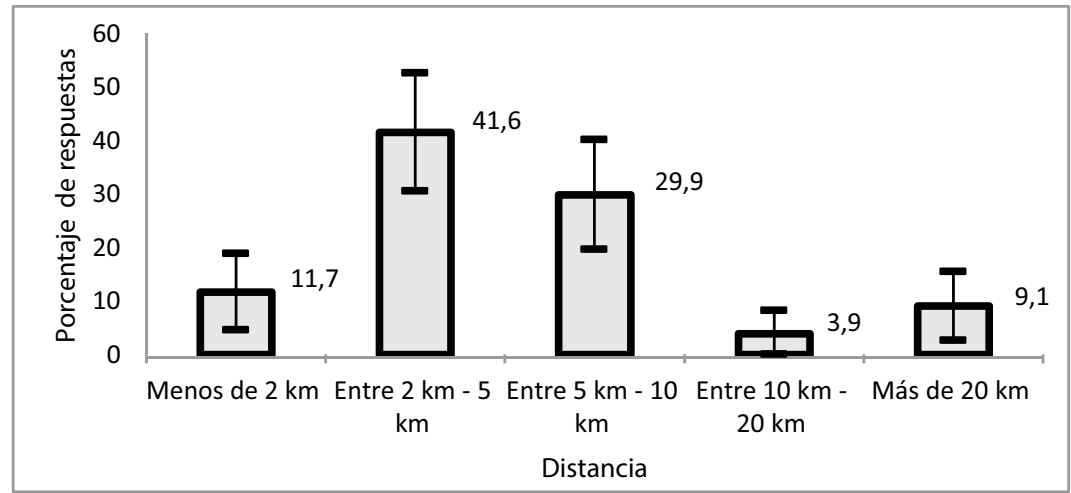

Figura 1. Universidad de Costa Rica. Distancia de viaje desde el lugar de residencia. Las barras de error indican los intervalos de confianza al $95 \%$. Fuente: elaboración propia con datos de encuesta 2013. 
Se trazó cada una de las rutas descritas por quienes son usuarios de bicicletas en sus recorridos típicos para viajar a la Universidad y a partir de las coincidencias entre ellas se obtuvieron la cantidad de personas usuarias por tramos (ver Figura 2). Los tramos con mayor cantidad de viajes incluyen la vía principal de Tibás (Ruta Nacional 102), la vía entre Moravia y Guadalupe (Ruta Nacional 109), la calle entre Guadalupe y Betania (Ruta Nacional 201) y la vía principal de Curridabat (Ruta Nacional 2).

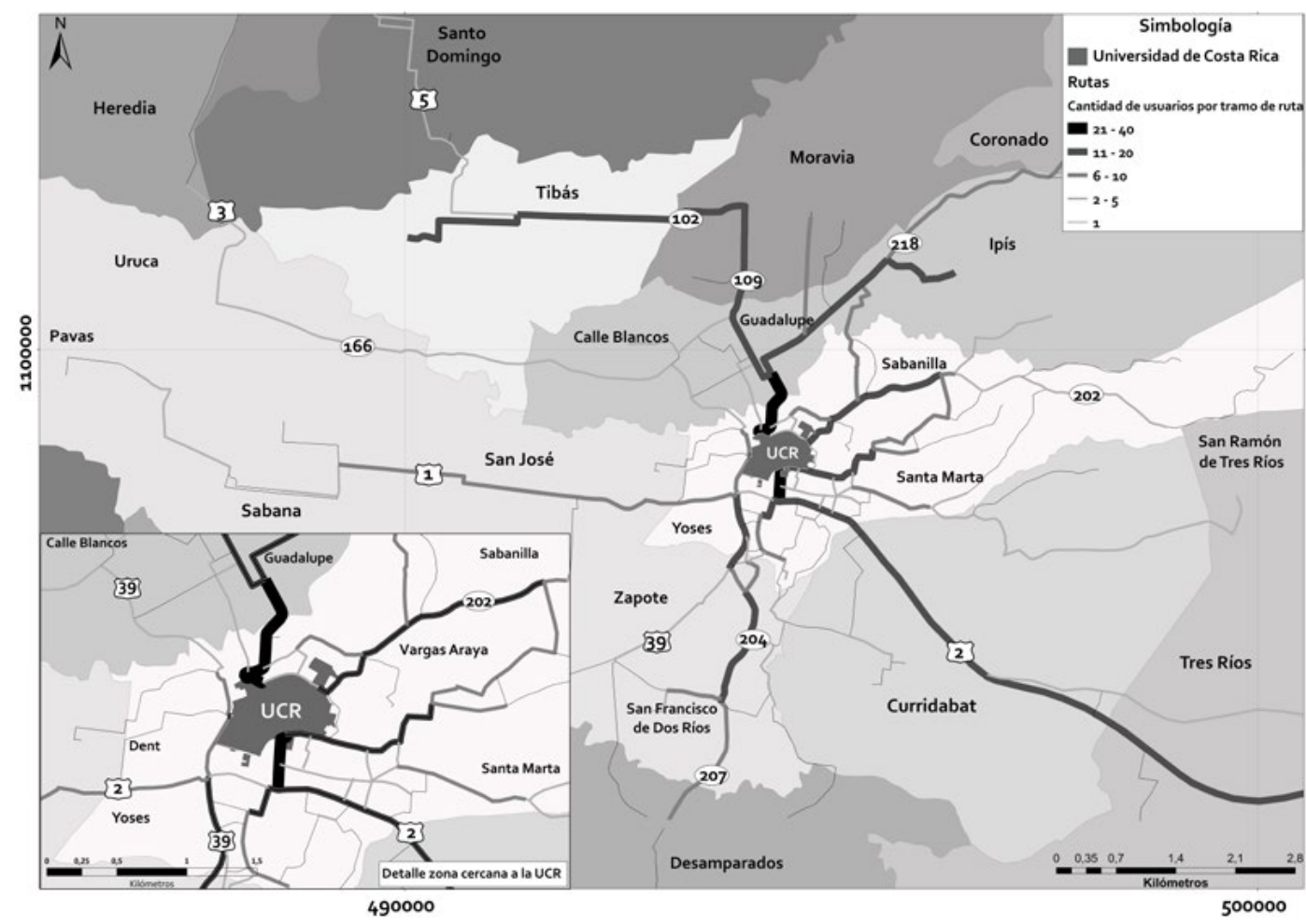

Figura 2. Mapa de rutas descritas por los ciclistas en la encuesta. Los tonos y espesores de líneas muestran la cantidad de usuarios por tramo de ruta. Fuente: Elaboración propia. 


\section{Caracterización de la movilidad en bicicleta}

De acuerdo con los intervalos de confianza mostrados en la Figura 3, la existencia de carriles exclusivos para ciclistas, espacios para parqueo en el lugar de destino y educación para los conductores resaltan como las razones que más motivan el uso de la bicicleta, respecto a las últimas siete opciones mostradas en la figura.

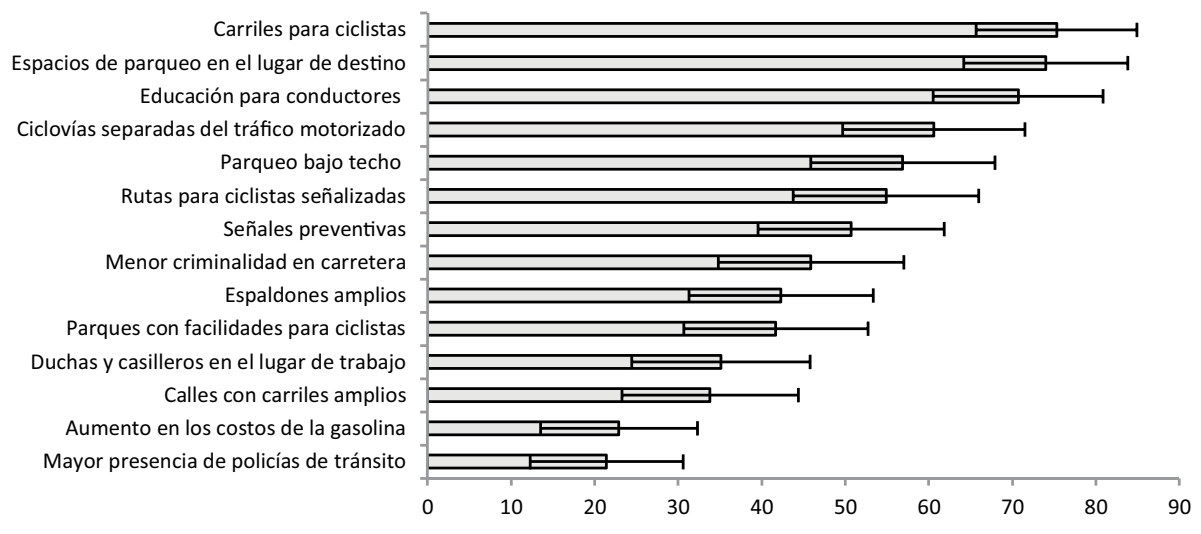

Figura 3. Principales aspectos que motivan extremamente el uso de la bicicleta. Las barras de error indican los intervalos de confianza al $95 \%$. Fuente: elaboración propia con datos de encuesta 2013.

Las calles con tráfico a alta velocidad, la falta de iluminación durante la noche y la presencia de vehículos pesados son aspectos que generan mayores sensaciones de inseguridad al utilizar la bicicleta para transportarse.

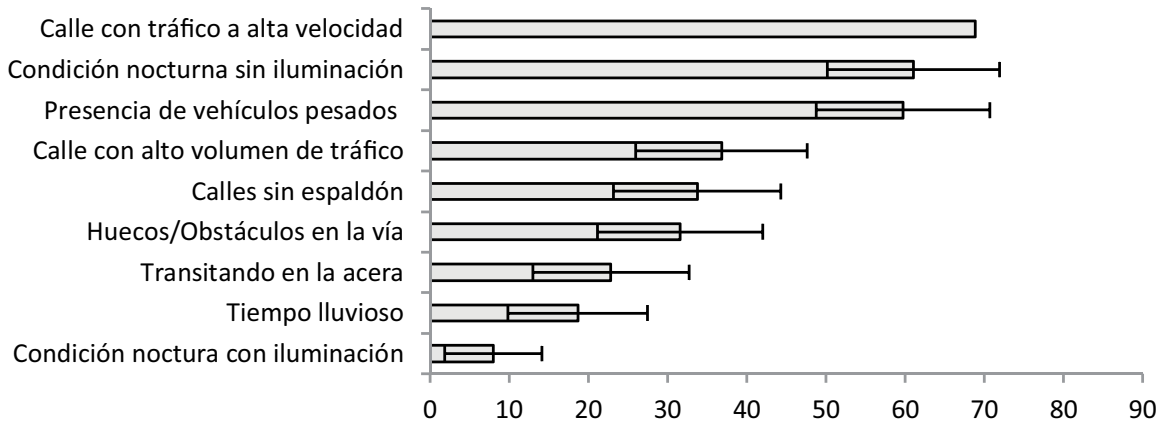

Figura 4. Percepción de la seguridad de los usuarios de bicicleta ante diferentes situaciones que se presentan en ruta. Las barras de error indican los intervalos de confianza al $95 \%$. Fuente: elaboración propia con datos de encuesta 2013. 
La ausencia de carriles para bicicletas y el hecho de que quienes conducen automóviles no compartan la vía son identificadas como aspectos problemáticos que dificultan el uso de la bicicleta. Otros elementos identificados destacan en la Figura 5 dado que existe un traslape en los intervalos de confianza en la mayoría de los casos.

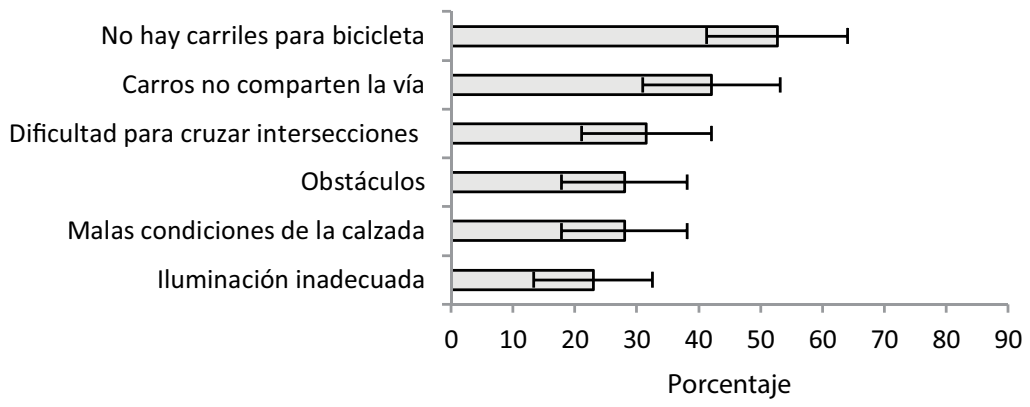

Figura 5. Situaciones calificadas como problemas que siempre afectan usuarios de bicicleta durante su traslado. Las barras de error indican los intervalos de confianza al 95 \%. Fuente: elaboración propia con datos de encuesta 2013.

Sobre las situaciones más desalentadoras para el traslado en bicicleta, la falta de lugares de parqueo $(74,6 \pm 10,1 \%)$, y las condiciones inseguras de la vía $(67,6 \pm 10,7 \%)$, se ubican entre los principales aspectos citados. Sin embargo, existe un traslape en varios intervalos de confianza tal como se muestra en la Figura 6.

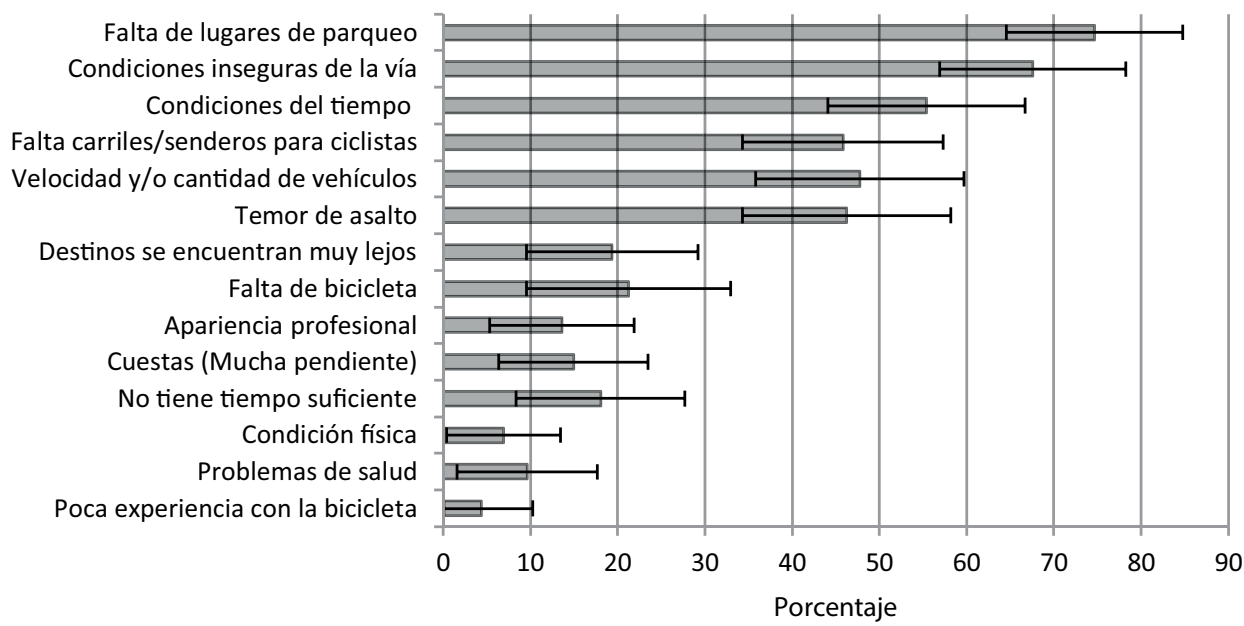

Figura 6. Aspectos que desalientan el uso de la bicicleta. Las barras de error indican los intervalos de confianza al 95 \%. Fuente: elaboración propia con datos de encuesta 2013. 


\section{Discusión}

"La inactividad física constituye en la mayoría de las regiones del mundo un importante problema de salud pública, cuya solución no parece pasar meramente por la adopción de enfoques tradicionales de promoción de la salud. El fomento del transporte activo (en bicicleta y a pie) para la actividad física diaria es una medida que resulta beneficiosa para todos, ya que, al mismo tiempo que promueve la salud, puede tener efectos ambientales positivos, sobre todo si la práctica de montar en bicicleta o caminar se realiza en sustitución de trayectos cortos en coche" (Organización Mundial de la Salud, 2014).

Los datos del estudio realizado muestran que solo un uno por ciento de los viajes que se realizan hacia la sede Rodrigo Facio son en bicicleta (Hernández y Jiménez, 2014); este porcentaje es el mismo que el reportado por Dobles (1981) en un estudio de los años ochenta, esto indica que el porcentaje de ciclistas no ha variado a través de los años, lo que puede ser un reflejo de la ausencia de políticas integrales para el fomento del uso de la bicicleta dentro y fuera de la universidad, anterior a estas nuevas políticas recientes. Por ejemplo, los carriles para ciclistas se encuentran dentro de los principales motivadores para el uso de la bicicleta; sin embargo, al momento de recopilar la información no existía un carril exclusivo para ciclistas en un radio de 10 kilómetros alrededor del Campus a pesar de que la mayoría de los viajes en bicicleta hacia el campus se realiza en distancias menores a ese tramo. La implementación de parqueaderos para bicicletas en el Campus se implementó en el 2014 un año después de la recopilación de la información. De acuerdo con los datos del sondeo, es prioritaria la construcción de carriles exclusivos, programas de pacificación del tránsito que logren una disminución de la velocidad del tránsito junto con programas de educación a conductores de la vía, entre otros.

De acuerdo con una minuciosa revisión de múltiples intervenciones para fomentar el uso de la bicicleta realizada por Pucher, Dill \& Handy (2010), se concluye que las políticas públicas cumplen un rol fundamental para la promoción del uso de ese medio de transporte y que para lograr un incremento significativo en el uso del mismo es necesaria la implementación de un paquete exhaustivo e integral de intervenciones que van desde la construcción de infraestructura ciclista hasta programas de educación y promoción.

Caminar y movilizarse en bicicleta como parte de las actividades diarias debe convertirse en un pilar importante de la estrategia para aumentar los niveles de actividad física como parte de la reducción de factores de riesgo en la salud de la población. El incremento de transporte no motorizado también reducirá la contaminación del aire y el ruido y mejoraría la calidad de vida urbana (Organización Mundial de la Salud, 2002).

La Organización Mundial de la Salud (2009), indica que solamente una tercera parte de los países del mundo tiene políticas que promuevan la movilidad activa y casi la mitad de los países 
no tienen políticas que incentiven el uso de transporte público. También menciona que estos datos podrían reflejar el desinterés de los gobiernos respecto a los usuarios de las vías que no utilizan vehículos motorizados.

La promoción del uso de medios de transporte no motorizado debe tomar en cuenta el tema del género, ya que estudios demuestran que ha sido identificado como un importante indicador en los patrones de viaje (Law, 1999). Las mujeres, además de realizar viajes con distancias más cortas que los hombres, utilizan menos la bicicleta. Figueroa y Waintrub (2015), muestran que el porcentaje de hombres que utilizan la bicicleta es mayor al porcentaje de mujeres que la utilizan como medio de transporte. Este aspecto resalta en el sondeo donde solo un $(19,5 \pm 8,8$ $\%)$ de los viajes en bicicleta hacia la UCR lo realizan mujeres.

Respecto a las distancias recorridas, los resultados obtenidos indican que la mayor cantidad de viajes corresponden a lugares relativamente cercanos, entre los 2 y los 10 kilómetros de distancia, con una mayor tendencia entre los 2 y 5 kilómetros. Estos datos coinciden con estudios que se han realizado en diferentes regiones, tal es el caso del realizado por Moritz (1997) donde al menos la mitad de los viajes en bicicleta en Norteamérica son de 5 kilómetros o menos. Los resultados del sondeo permiten suponer que la distancia recorrida por los ciclistas universitarios podría ser similar, a la distancia reportada en estudios en otros países. Estos resultados son útiles para definir las zonas de intervención de la infraestructura en los alrededores del campus.

"La bicicleta se constituye como un potencial medio de transporte en los centros urbanos, principalmente para la realización de viajes cortos o complementarios con el transporte público" (Cámara de Comercio de Bogotá, 2009, p. 10). Un reporte sobre el ciclismo en Dinamarca muestra, que el incremento de los viajes en bicicleta entre el 2007 y el 2012 ha sido aproximadamente del $9 \%$ y de estos viajes la mayor concentración se da en viajes entre los 2 y 5 kilómetros, coincidiendo con los datos mostrados la Figura 1. Además, resaltan los viajes entre los 5 y los 10 kilómetros y se refleja una diferencia ya que los viajes cortos de menos de 2 kilómetros tienen una importancia mayor (Centre for Regional Development of Denmark, 2015).

Los resultados mostraron que las problemáticas que enfrentan los ciclistas y las ciclistas, los aspectos que los motivan y los desalientan a utilizar este medio de transporte, están estrechamente relacionados y sobresalen: la infraestructura disponible (carriles, parqueos), la educación (automóviles no comparten la vía) y las condiciones inseguras. Estos aspectos deben considerarse a la hora de plantear en la promoción del uso de medios de transporte no motorizados.

Kumar, Meng Teo \& Odoni (2012), plantean que la seguridad juega un papel clave en la toma de la bicicleta como una opción creíble, en un entorno urbano de tráfico mixto. A medida que el nivel de seguridad mejore, más viajeros optarán por usar la bicicleta. De acuerdo con los 
resultados del presente estudio se percibe una mayor inseguridad en las calles con tráfico a alta velocidad, así como en lugares con falta de iluminación en las noches.

De igual forma, la disponibilidad de carriles-bici está asociada con tasas más altas de desplazamientos en bicicleta, una red de diferentes tipos de infraestructura resulta necesaria para atraer a nuevas personas para utilizar la bicicleta; para personas preocupadas por la seguridad y evitar el tráfico, una red bien conectada de calles de poco tráfico, incluyendo algunos bulevares bici, puede resultar más efectiva que la adición de carriles-bici en las principales calles con altos volúmenes de tráfico de vehículos motorizados (Dill, 2009).

Según Pucher y Dijkstra (2003), para el año 1995 en Estados Unidos se realizaban cerca de $6 \%$ de los viajes de forma no motorizada mientras que en países europeos como Holanda y Dinamarca estos viajes superaban el $40 \%$. Los países europeos que tienen los niveles más altos de viajes en bicicleta o a pie, tienen menores tasas de obesidad, diabetes e hipertensión que Estados Unidos. Países desarrollados en donde se realizan gran parte de los viajes de manera activa, no motorizada, tienen una esperanza de vida de entre 2,5 y 4,4 años más que en Estados Unidos y los gastos per cápita en salud son de la mitad. Pese a que es claro que son muchos los factores que influyen en estas diferencias, estudios muestran que los dramáticos niveles más altos de viajes caminando y en bicicleta diariamente sin duda contribuyen a mejorar la salud pública de los países (Pucher \& Dijkstra, 2003). Se recomienda que se genere en Costa Rica información sobre el efecto del uso de la bicicleta en la salud. Por otro lado, en la Política Nacional de Salud (Gobierno de la República de Costa Rica, 2015) se considera a las formas de transporte activas como actividad física (pág. 114) y su promoción se encuentra dentro de las estrategias incluidas en el plan.

La actividad física es asociada con un mejor bienestar subjetivo, estado de ánimo y de las emociones, de igual forma puede mejorar la autoestima y puede resultar en cambios positivos en aspectos relacionados con la auto percepción de la imagen corporal. Las personas activas también reportan menos síntomas relacionados con ansiedad, angustia o estrés emocional y presentan mejoras en los patrones del sueño (Cavil \& Davis, 2007).

Es posible que las ciudades que promuevan el transporte no motorizado se vean beneficiadas económicamente con un aumento en la oferta de trabajos, el fortalecimiento del comercio local con la implementación de corredores comerciales, y también con una disminución en los costos por la atención a accidentes (Simmons et al, 2015). 


\section{Conclusiones}

El sondeo realizado muestra datos que permiten mejorar la comprensión sobre la movilidad en la bicicleta como medio de transporte en la Universidad de Costa Rica. Esta información aporta nuevos elementos para la gestión de políticas en el mediano y largo plazo que promuevan el aumento de la cantidad de personas que acuden a la universidad utilizando la bicicleta como medio de transporte.

El bajo porcentaje de mujeres que utiliza la bicicleta llama a la reflexión con respecto al tema del sexo y movilidad, en donde además, el tiempo y la distancia de viaje juegan un papel central en cuanto al uso de la bicicleta. Acuña (2015), sugiere que la existencia de infraestructura ciclista podría promover un mayor número de mujeres utilizando la bicicleta.

Producto de los resultados obtenidos y de su análisis, es posible realizar recomendaciones que con su aplicación puedan cumplir con los objetivos de tener un campus universitario más saludable, en el cual la bicicleta forme parte importante y sea vista como medio de transporte habitual, de manera que se logre disfrutar de sus beneficios.

Estos beneficios resultan cuantificables una vez que se aumente el uso de la bicicleta por parte de las personas que visitan la sede universitaria. Promover rutas de baja velocidad y la construcción de infraestructura es fundamental para la promoción del ciclismo urbano, si se toma en cuenta que desde los resultados obtenidos por Dobles (1981), la construcción de parqueos y la implementación de infraestructura vial para ciclistas, formaban parte de las principales opciones para lograr promover un mayor uso de la bicicleta.

El alto uso observado de la mayoría de los aproximadamente 100 espacios de parqueo para bicicletas construidos posteriormente a la recopilación de la información en 21 lugares en el campus, viene a responder a las necesidades de los usuarios y a la vez viene a justificar no solo la instalación de más ciclo estacionamientos, sino también el desarrollo de programas permanentes de control y evaluación.

La sencillez de movilidad es un aspecto central a la hora de tomar la decisión de utilizar la bicicleta, pero el tema de la percepción de seguridad es fundamental para que las personas continúen utilizándola y a la vez se aumente la cantidad de usuarios. Aspectos que influyen en la sensación de inseguridad son la velocidad de los vehículos automotores, la presencia de vehículos pesados y la conducción nocturna sin iluminación víal.

Es necesario ver esta información a un nivel de escala de intervención mayor en el desarrollo de políticas, como la generación de un plan de movilidad en bicicleta para el campus y las zonas aledañas identificadas como prioritarias, esto en conjunto con las municipalidades vecinas y el 
Ministerio de Obras Públicas y Transportes. Respecto a las acciones necesarias para implementar el plan de movilidad en bicicleta se sugiere considerar las recomendaciones indicadas por ITDP (2011) para la generación de políticas públicas y planes de movilidad, y sustentar las decisiones en la revisión de Pucher, Dill \& Handy (2010) sobre los diferentes paquetes de intervenciones implementadas en diferentes ciudades alrededor del mundo a la luz de los resultados de la caracterización de movilidad ciclista mostrados en este estudio.

Un tema adicional a los aspectos de inseguridad ya anotados, es que las principales rutas de vehículos pesados, como la Ruta Nacional 2 también son rutas escogidas por los ciclistas y las ciclistas, aspecto que debe ser evaluado por las autoridades correspondientes.

Estudios de movilidad en bicicleta permiten conocer las características y percepciones de la población de ciclistas, y a su vez se convierte en una herramienta para dimensionar mejoras y medidas que permitan incentivar el uso de la bicicleta. La implementación de planes que promuevan la movilidad activa, requieren de estudios previos con el fin de generar procesos donde la toma de decisiones se realice de una manera sustentada.

\section{Agradecimientos}

Se agradece la colaboración del Centro de Informática de la UCR que brindó asesoría y capacitación sobre el programa utilizado para la realización del cuestionario electrónico y la colaboración de la Oficina de Divulgación e Información (ODI) y la Oficina de Registro e Información (ORI) en la difusión del instrumento. 


\section{Referencias bibliográficas}

Acuña, R. (2015). Desarrollo de una metodología para el diseño y la evaluación de ciclovías en Costa Rica. (Trabajo Final de Graduación para obtener el grado de Licenciatura en Ingeniería Civil). Universidad de Costa Rica.

Alta Planning + Design; Kimley-Horn and Associates, Inc. (2012). University of Arizona Area Bicycle and Pedestrian Plan. Pima Association of Governments and University of Arizona. Recuperado de https://parking.arizona.edu/alternative/documents/UAAreaBikePedPlanFinalAugust2012. pdf

Balsas, C. (2003). Sustainable transportation planning on college campuses. Transport Policy, 10(1), 35-40. doi:10.1016/S0967-070X(02)00028-8

Banco Interamericano de Desarrollo (2015). Ciclo-inclusión en América Latina y el Caribe. Guía para impulsar el uso de la bicicleta.

Burgos, N. y Marín, A. (2014). UCR busca convertirse en campus saludable. Recuperado de http:// www.ucr.ac.cr/noticias/2014/03/07/ucr-busca-convertirse-en-campus-saludable.html

Cámara de Comercio de Bogotá (2009). Movilidad en Bicicleta en Bogotá. Bogotá, Colombia.

Cavill, N \& Davis, A. (2007). Cycling \& Health. What's the evidence? Cycling England.

Centre for Regional Development of Denmark. (2015). Capital Region of Denmark: Regional Cycling Report. Dinamarca. Recuperado de http://www.cycling-embassy.dk/wp-content/ uploads/2015/03/17751-Cykelregnskab_UK.pdf

Cuddeback, G., Wilson, E., Orme, J. G., \& Combs-Orme, T. (2004). Detecting and statistically correcting sample selection bias. Journal of Social Service Research, 30(3), 19-33.

Díaz de Rada, V. (2012). Ventajas e inconvenientes de la encuesta por Internet. Revista Papers, 97(1), 193-223.

Dickinson, J., Kingham, S., Copsey, S., y Pearlman, D. (2003). Employer travel plans, cycling and gender: will travel plan measures improve the outlook for cycling to work in the UK? Transportation Research, 53-67

Dill, J. (2009). Bicycling for Transportation and Health: The Role of Infrastructure. Journal of Public Health Policy. 30, 95-110. doi:10.1057/jphp.2008.56 
Dobles, M. (1981). Circulación de bicicleta en el Área Metropolitana. Proyecto de Investigación. Escuela de Ingeniería Civil. Universidad de Costa Rica.

Duncan, S., Duncan, E., Fernandes, R., Buonani, C., Bastos, K., Segatto, A., Codogno, J., Gomes, I. y Freitas, I. (2011). Modifiable risk factors for overweight and obesity in children and adolescents from São Paulo, Brazil. BMC Public Health. 11(1), 585. Recuperado de http://www.biomedcentral. com/1471-2458/11/585\#

Espinoza, J. (2015). Estudio de movilidad ciclista en los distritos del cantón de Puntarenas con mayor cantidad de ciclistas involucrados en colisiones. (Trabajo Final de Graduación para obtener el grado de Licenciatura en Ingeniería Civil). Universidad de Costa Rica.

Esquivel, A., González, R., y Martínez, E. (2012). Contraste de las preferencias declaradas con las preferencias reveladas: El caso de los alumnos de la Universidad de la Laguna ante la implementación del Tranvía. Metodología de encuestas, 14, 65-80

Figueroa, C., y Waintrub, N. (2015). Movilidad femenina en Santiago de Chile: reproducción de inequidades en la metrópolis, el barrio y el espacio público. urbe. Revista Brasileira de Gestão Urbana, 7(1), 48-61

Forsman, Å., Gustafsson, S., \& Vadeby, A. (2007). Impact of Nonresponse and Weighting in Swedish Travel Survey. Transportation Research Record: Journal of the Transportation Research Board, (1993), 80-88.

Gobierno de la República de Costa Rica (2015). Política Nacional de Salud “Dr. Juan Guillermo Ortiz Guier". Recuperado de https://www.ministeriodesalud.go.cr/index.php/biblioteca-dearchivos/sobre-el-ministerio/politcas-y-planes-en-salud/politicas-en-salud/2746-politicanacional-de-salud-2015/file

Gotschi, T. (2011). Costs and Benefits of Bicycling Investments in Portland, Oregon. Journal of Physical Activity and Health, Vol. 8(1), 49-58

Heerwegh, D. y Loosveldt, G. (2009). Face to face versus web surveying in a high internet coverage population. Public Opinion Quarterly, 72(5), 836-846

Hernández, A. y Jiménez, D. (2014). Informe No. LM-PI-USVT-006-14. Resultados de la encuesta de transporte. Sede Rodrigo Facio. Laboratorio Nacional de Materiales y Modelos Estructurales, Universidad de Costa Rica. San Pedro de Montes de Oca, Costa Rica. 
ITDP (2011) Manual Ciclociudades. Instituto para Políticas de Transporte y Desarrollo, México México, DF. Disponible en: http://ciclociudades.mx/manual-ciclociudades/

Kaplan, D. (2015). Transportation sustainability on a university campus. International Journal of Sustainability in Higher Education, 16(2), 173-186.

Kumar, A., Meng Teo, K., \& Odoni, A. (2012). A Systems Perspectives of Cycling and Bike-sharing Systems in Urban Mobility. 30th International Conference of the Systems Dynamics Society. St. Gallen, Switzerland. Recuperado de http://www.systemdynamics.org/conferences/2012/ proceed/papers/P1306.pdf

Law, R. (1999). Beyond 'women and transport': towards new geographies of gender and daily mobility. Progress in Human Geography, 23(4), 567-588

Leandro, M., Molina, O., \& Riba, L. (2013). Intersecciones de la movilidad activa: Construcción interdisciplinar del espacio público en el campus universitario. On the w@terfront, 28, 40-56

Loría, T. (2014). Caracterización de los estilos de vida, nivel de actividad física y prevalencia de obesidad en la población estudiantil de la Sede de Occidente, para la Universidad de Costa Rica: Aporte para un plan de intervención. (Tesis de Posgrado en Salud Integral y Movimiento Humano). Universidad de Nacional. Heredia, Costa Rica.

Lynch, G., \& Atkins, S. (1988). The influence of personal security fears on women's travel patterns. Transportation, 15(3), 257-277.

Mackett, R. L., Lucas, L., Paskins, J., \& Turbin, J. (2005). The therapeutic value of children's everyday travel. Transportation Research Part A: Policy and Practice, 39(2), 205-219

Medina, S. y Veloz, J. (2013). Desarrollo Orientado al Transporte. Instituto de Políticas para el Transporte y el Desarrollo México. Recuperado de http://mexico.itdp.org/wp- content/ uploads/Desarrollo-Orientado-al-Transporte.pdf

Millard-Ball, A., Siegman, P. \& Tumlin, J. (2004), Solving campus parking shortages: new solutions for an old problem. Planning for Higher Education, 33(1), 30-43

Ministerio de Salud (2010). Plan Nacional de Actividad Física y Salud 2011-2021. Recuperado de https://www.ministeriodesalud.go.cr/index.php/sobre-ministerio/politcas-y-planes-ensalud/planes-en-salud/1 164-plan-nacional-de-actividad-fisica-y-salud-2011-2021/file 
Moritz, W. (1997). Survey of North American Bicycle Commuters. Desing and Aggregate Results. Transportation Research Record: Journal of the Transportation Research Board, (1578), 91-101

Organización Mundial de la Salud (2017). Actividad física. Recuperado de http://www.who.int/ mediacentre/factsheets/fs385/es/

Organización Mundial de la Salud (2014). Herramientas de evaluación económica de la salud (HEAT) para los desplazamientos a pie y en bicicleta. Ginebra. Recuperado de http://www.who.int/ dietphysicalactivity/publications/HEAT_walking_cycling/es/

Organización Mundial de la Salud (2009). Informe sobre la situación mundial de la seguridad vial: es hora de pasar la acción. Ginebra. Recuperado de http://www.who.int/violence_injury_ prevention/road_safety_status/report/web_version_es.pdf?ua=1

Organización Mundial de la Salud (2002). Physically active life through everyday transport. World Health Organization Regional Office for Europe, Copenhague, Dinamarca.

Organización Panamericana de la Salud (2005). Políticas municipales para la promoción de la actividad física: Diálogo entre salud, transporte, obras públicas y seguridad pública. Lima, Perú.

Portland State University (2011). The Bicycle Transportation Plan. Recuperado de https:/www.pdx. edu/campus-planning/sites/www.pdx.edu.campus-planning/files/PortlandStateUniversity_ BicycleTransportationPlan_March2011_ScreenRes.pdf

PRUGAM (2008). Plan Regional Urbano de la Gran Área Metropolitana de Costa Rica. Tomo I: Diagnóstico Plan PRUGAM 2008-2030. Recuperado de http://exnet.mivah.go.cr/PRUGAM/ Documentos/Diagnostico.pdf

Pucher, J. \& Dijkstra, L. (2003). Promoting sake walking and cycling to improve public health: Lessons from the Netherlands and Germany. American Journal of Public Health, 93 (9), 1509-1516.

Pucher, J., Dill, J., \& Handy, S. (2010). Infrastructure, programs, and policies to increase bicycling: an international review. Preventive medicine, 50, S106-S125.

Rodríguez, Á., Urbaneta, E., de la Fuente, M., Galindo, E., Yanguas, J., \& Rodríguez, V. (2013). Análisis del sesgo de selección en el piloto de un estudio longitudinal sobre envejecimiento en España. Gaceta Sanitaria, 27(5), 425-432. 
Simmons, E., Kay, M., Ingles, A., Khurana, M., Sulmont, M. \& Lyons, W. (2015). Evaluating the Economic Benefits of Nonmotorized Transportation: Case Studies and Methods for the Nonmotorized Transportation Pilot Program Communities (No. DOT-VNTSC-FHWA-14-03). US Department of Transportation. Recuperado de http://www.pedbikeinfo.org/cms/downloads/NTPP Economic_Benefits_White_Paper.pdf

Toor, W. and Havlick, S. (2004), Transportation and Sustainable Campus Communities: Issues, Examples, Solutions, Island Press, Washington, DC.

Torres, M. (2016). Estudio de movilidad ciclista en los distritos de San Francisco, Guadalupe y quebradilla del cantón de Cartago, y los distritos de Tobosi y Tejar del cantón de El Guarco. (Trabajo Final de Graduación para obtener el grado de Licenciatura en Ingeniería Civil). Universidad de Costa Rica.

Turner, T; y Niemeier, D. (1997). Travel to work and household responsibility: new evidence. Transportation, 24(4), 397-14

Universidad de Costa Rica (2012). Plan de mejoramiento para la movilidad y el transporte en la Ciudad Universitaria. Recuperado de http://www.ucr.ac.cr/plan-mejoramiento-movilidad.html

University of California Berkeley (2006). Campus Bicycle Plan. Recuperado de http://pt.berkeley.edu/ sites/default/files/UCB_BikePlanFinal.pdf

University of North Carolina at Greensboro (2008). Campus Bicycle Master Plan. Recuperado de http://parking.uncg.edu/docs/UNCGBicycleMasterPlanFullFINAL.pdf

Vives, A., Ferreccio, C., \& Marshall, G. (2009). Comparación de dos métodos para corregir el sesgo de no respuesta a una encuesta: sustitución muestral y ajuste según propensión a responder. Gaceta Sanitaria, 23(4), 266-271.

Wen, L. \& Rissel, C. (2008). Inverse associations between cycling to work, public transport, and overweight and obesity: Findings from a population based study in Australia. Preventive Medicine, 46(1), 29-32. doi:10.1016/j.ypmed.2007.08.009

Wen, L., Orr, N., Millett, C. y Rissel, C. (2006). Driving to work and overweight and obesity: findings from the 2003 New South Wales Health Survey, Australia. International Journal of Obesity, 30(5), 782-786. Recuperado de http://www.nature.com/ijo/journal/v30/n5/pdf/0803199a. pdf 